ARIZONA HEALTH FUTURES

Policy Primers: a nonpartisan

guide to a better understanding

of key terms and issues in the

Arizona health policy landscape.

\title{
Can This Marriage Be Saved? Physician-Hospital Relationships
}

ITEM: D.S. is an orthopedic surgeon. He does most of his work in a state-of-the-art ambulatory surgery center, where he has more control over his schedule - and sees paying patients who have insurance. "It's not that I didn't like working in the hospital," he explained. "It's that I don't need to anymore."

ITEM: F.B. is an internist. In the late 1990s he grew tired of HMOs telling him whom he could and couldn't see in the hospital, so he and other physicians formed a local hospitalist group and contracted exclusively with a Valley hospital to provide inpatient services. "I wasn't in charge in the outpatient setting," he said. "In the hospital I treat more patients, have fewer administrative hassles, and get to practice medicine the way I was trained to do. It's like being part of a family."

Two different physicians, two stories and one overarching issue: the market forces in American health care that paradoxically are pulling physicians and hospitals apart and together at once.

Every hospital needs physicians, but not every physician needs hospitals. Or do they?

What are the forces and trends in health care that impact physicianhospital relationships? Is their long-standing marriage of interdependence and productivity destined to fail, or can it be saved and even strengthened by emerging delivery and governance models in the so-called "market revolution" of consumer-driven health care? What are the implications for health care policy and practice?

These are issues we explore in this Arizona Health Futures Policy Primer.

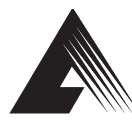

Arizona Hospital and Healthcare Association

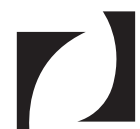

St. Luke's Health Initiatives

A Catalyst for Community Health 


\section{An SLHI-AzHHA Collaboration}

This AHF Policy Primer is a collaborative effort between SLHI and the Arizona Hospital and Healthcare Association's 2005 Building the Bench Leadership Development Program. As part of their educational activities, the 17 participants from 13 Arizona hospitals were asked to break into teams and each prepare a white paper on the issue of Changing Relations Between Hospitals and Physicians. AzHHA asked SLHI to use the white papers as the basis for a background report to inform Arizona policy leaders on the implications of changing physician-hospital relations for issues of health care access, quality and cost.

In addition to utilizing the work of the Building the Bench hospital executives, SLHI also conducted further background research, including interviews with practicing physicians in Arizona.

\section{Background}

The rocky marriage between physicians and hospitals is hardly a new phenomenon. The tension between the concept of the hospital as the "doctor's workshop," subject to medical control, and the hospital as an independent corporation with its own locus of control and set of functions has waxed and waned for most of the twentieth century. ${ }^{1}$

Beginning with the control of workmen's compensation funds by enterprising hospital administrators around 1915 and accusations by physicians that hospitals were selling their charitable services for a profit, ${ }^{2}$ the struggle for autonomy, control, respect, power and money has proceeded apace. Physicians, trained and enshrined as the arbiters of all medical practice, viewed with alarm the increasing portion of the nation's health care dollar flowing directly to hospitals, and not to them. In turn, a new breed of non-physician hospital administrators, trained to run their institutions as a business, viewed physicians as resentful of "lay" control and the rules and regulations necessary to run a sound and efficient enterprise. With no small amount of understatement, one administrator remarked in 1956, that "...doctors, because they are doctors, are hard to fit into hospital organization."

And so it has gone. There have been periods of cooperation, particularly in the face of a perceived common "enemy" such as third party payers and oppressive government regulation and payment mechanisms, but by and large the "provider" community of hospitals and physicians has remained a contentious lot for much of the twentieth century. Although the daily dealings of physicians and hospitals appeared outwardly cordial, there was often "smoldering distrust, antagonism, resentment and even hatred" between the two groups. ${ }^{4}$

The agenda of physicians was to control the scope of independent, private practice with minimal barriers between themselves and their patients. The ethos of the hospital medical staff, the dominant model of physician-hospital affiliation, was maximum physician autonomy and minimum "interference" from so-called standardized hospital procedures. The agenda of the increasingly rationalized "corporate" hospital, on the other hand, was to increase the efficiency of medical production in order to realize ever greater gains to the financial bottom line - the charity mission of voluntary nonprofit hospitals and the real desire to improve health outcomes notwithstanding. ${ }^{5}$ The marriage was one of necessity - each needed the other - but it was not without competing interests and discord. 


\section{The Old is New Again}

Not all physicians and hospitals, of course, are the same. The marriage can be quite different for physicians employed in large integrated hospital settings, for certain medical specialties that are primarily found in hospitals, for selected joint hospital-physician medical enterprises, and for hospitals that assiduously cultivate mutually productive relationships with physicians. We note some of the ingredients of these strong marriages as we proceed. The general point, however, is that physician-hospital tensions have been evident ever since the genesis of the "industrialization" of American health care in the early twentieth century. It's safe to predict that they are not going to go away anytime soon.

Like most of the issues that constitute today's chronic "crisis" in health care, the old is suddenly new again.

\section{Historical Eras of Affiliation ${ }^{6}$}

Hospitals can't write an order, perform a procedure, meet organizational goals and investor expectations, or achieve their mission of community health without physicians. On the other side, physicians need hospitals for access to capital and technology, for an operational "theater" in which to practice their craft, for efficiency and effectiveness of care, and for the education and training of future physicians and other health care professionals.

Beyond this basic interdependency, hospitals might seek to strengthen their affiliation with physicians for one or more of four reasons: ${ }^{7}$

1. COORDINATION OF CARE. Important when hospitals receive additional income for controlling health care costs and improving quality.

2. LEVERAGE WITH HEALTH PLANS. Important when bargaining with health plans that contract selectively with certain hospitals or networks.

3. LEVERAGE ADMISSIONS. Always important, but especially during times of increased competition, tight margins and under-utilized capacity.

4. RISK SHARING. Important "when Health Maintenance Organizations (HMOs) pass on all or part of the financial risk for the costs of patient care to physicians and/or hospitals."

Researchers posit three "eras" of medical care with consequences for the strength of physician-hospital affiliation:

1. PRE-MANAGED CARE. This covers most of the twentieth century. Fee-for-service constitutes the payment landscape, procedures increase, controlling cost of care is less important, quality isn't measured. The medical staff model - loose affiliation and maximum physician autonomy/control - is dominant.

2. TIGHT MANAGED CARE. Mid-1980s and early 1990s. Rise of the tightly managed HMO, capitation, case and per diem rates; selective contracting, gatekeeper requirements. Competition for physician affiliation intensifies. Tighter physician affiliation with Independent Practice Associations (IPAs) and Physician Hospital Organizations (PHOs).

3. LOOSE MANAGED CARE. Late 1990s - current. Backlash against tight managed care, risk contracting and gatekeeper requirements. However, still prevalence of negotiated rates, pressure to control costs, continuation of per diem payments, Medicare Prospective Payment System (PPS), continuity of care, quality outcomes, Pay-for-Performance $(\mathrm{P} 4 \mathrm{P})$. Reasons for hospitals to affiliate more tightly with physicians are weaker than in tight managed care era, but stronger than in pre-managed care era. 
WORK MORE, ENJOY IT LESS

In a 2002 Kaiser

Family Foundation

national survey of

2,308 physicians,

87 percent said

their overall morale

has declined during

the past five years.

Approximately 60

percent reported

their enthusiasm

for practicing

medicine has

dropped because

of managed care,

increased paper

work and practice

costs, less time

spent with patients,

limits placed on the

use of specialists,

less clinical

autonomy, and a

general feeling

that the quality

of health care is

being undermined. ${ }^{10}$
Generally, organizational literature suggests that "physicians have more incentive to be productive and have maximal flexibility to adjust their actions to changing conditions when they deal with one organization (in this case, hospitals) at arm's length [our italics] rather than be employed by an organization. However, the more tightly affiliated hybrid models or a tighter linkage in one firm/ownership may be advantageous when close coordination [our italics] is required."

What will it be as we move forward with hospital-physician affiliation in Arizona: Arm's length or closer coordination?

\section{Stress Factors}

Some of the stress factors that put physician-hospital relationships at risk: ${ }^{9}$

\section{HOSPITALS}

Weak financial reimbursement

Staffing shortages

Keeping up with technology

Increasing consumer expectations

Capacity constraints

Competition from niche

clinical providers

\section{PHYSICIANS}

Maintaining reasonable compensation

Maintaining clinical autonomy

Managing their business

Increasing consumer expectations

Malpractice premiums/

business overhead

Balance between professional and personal time

\section{External Factors Affecting Physician-Hospital Relationship}

Hospital administrators and physicians cite the following external factors as affecting current relations in Arizona:

\section{Physician Shortages}

Physician shortages - and shortages of skilled health professionals generally - have been noted for some time. Based on standard industry ratios, recent Arizona data indicates a shortfall of 1,566 physicians based on a population of 5.7 million. Projecting forward to a population of 6.4 million in 2009 , the shortfall is expected to be 2,693 physicians. ${ }^{11}$

The shortage impacts the hospitals' ability to recruit physicians and provide necessary services. This is particularly evident in selected specialties important to inpatient hospital and emergency care, such as surgery and orthopedics. It is also evident in locations perceived to be less attractive to some physicians, such as rural and low income areas.

Many physicians experience high stress with busy office practices, inpatient volumes that require physicians to make rounds, and discharge of hospital "on-call" responsibilities. In addition to prolonging or delaying patient care, shortages can also steer physicians away from hospital-based practice to group practice, where there is safety in numbers. Finally, shortages disrupt the "volunteerism" for time spent on hospital business, such as administrative and service functions. 
"It's the

This is the flip side of the supply-side shortage. Tensions between physicians and hospitals that are exacerbated in times of low demand are muted in times of increased demand.

Hospitals and physician services barely keep pace with Arizona's rapid population growth, especially in the fast-growing urban core of Maricopa, Pinal, Pima and Yuma counties.

Everyone is busy; hospital administrators and physician leaders worry about keeping their facilities and practices current with a torrent of demand.

To some extent, increased demand is stoked by competition for the most profitable services, such as diagnostic and imaging, selected surgical interventions and "lifestyle" alternatives. In Arizona, as elsewhere, more of these services are moving out of the hospital and into physicians' offices and the outpatient setting. In addition to making it harder for hospitals to underwrite less profitable "safety net" community services, it also induces demand for more expensive services and drives up health care costs - leading to more people unable to afford health insurance, etc. We return to this point later.

\section{Legal Considerations}

A number of legal considerations impact physician-hospital relationships:

- STARK SELF-REFERRAL STATUTE. The highly technical "Stark" law prohibits physicians from referring patients for certain categories of health services and items (e.g., radiology, hospital inpatient and outpatient services) with which the physician has an ownership or compensation relationship, unless certain exceptions apply. Stark Act compliance calls for hospitals to critically examine physician compensation/employment arrangements, leases for office space, equipment and services, and physician recruitment. Stark regulations limit physician entrepreneurial pursuits and can alter historical physician/ hospital compensation and expense sharing models. ${ }^{12}$

- ANTI-KICKBACK STATUTE. "Broader than Stark, this intent-based statute makes it a criminal felony to pay or receive anything of value in return for inducing referrals of federal health care program business. Certain arrangements fitting within a 'safe harbor' are guaranteed free from challenge." ${ }^{13}$ But not all arrangements between physicians and hospitals neatly fit into "safe harbors." Therein lie a number of thorny relationship issues.

- TAX-EXEMPTION STANDARDS. "If not consistent with tax-exemption standards, participation in a business arrangement with physicians can create monetary sanctions for a tax-exempt hospital and can even jeopardize the hospital's tax-exempt status."14 The problem comes in distinguishing between arrangements that "benefit the community" and those that are more directly focused on providing a for-profit physician group with a share of the hospital's revenue stream.

- EmtalA. The Emergency and Medical Treatment and Active Labor Act of 1986, better known as EMTALA, governs when and how the Centers for Medicare and Medicaid Services (CMS) - participating hospitals may refuse treatment to a patient, or how a patient is transferred from one hospital to another. While hospitals are required to provide services to stabilize patients in emergency settings, physicians may not be available to "take call." As more procedures and specialties migrate to outpatient settings, it becomes a challenge for the hospital to provide "mandated" care. 


\section{“Malpractice}

exposure in

the hospital

is substantial,

and the

reimbursement

can be lower

or even

nonexistent.

So why would

I want to go

there unless

I had to?"

Arizona Specialist
- feDERAL/STATE ANTITRUSt LAWS. A number of antitrust laws, including the Antitrust Healthcare Advancement Act of 1997, govern such issues as provider network collaboration, "collusion," price setting, financial soundness and other matters. These laws extend considerably beyond physician-hospital relations, but they complicate them nonetheless. One of the challenges of current efforts to improve system efficiency through more robust and integrated provider networks is dealing with the Stark and antitrust restrictions. Some believe these laws need to be overhauled.

\section{Reimbursement Issues}

Declining reimbursement (Balanced Budget Amendment, Medicaid/Medicare cuts, discounted fees, etc.) intersects with the twin trends of increased demand and ever higher operating expenses. This drives physicians to seek a larger share of the health care dollar through expansion into ambulatory surgery centers (ASCs), new health services "packages" in facilities external to hospitals, and a focus on "paying" populations, as distinct from uninsured and low-income persons who often end up in hospitals with complicated and expensive health problems.

Hospitals, meanwhile, are faced with the challenge of not only responding to broad community health needs (the uninsured, persons in need of immediate care) but also of continuing to invest in the technology and service infrastructure necessary to attract physicians and produce a healthy balance sheet. In only one of several major perversities of health care reimbursement practices, hospitals and physicians have historically been paid independently of each other, with neither payment based on how the other performed. For example, hospitals can be denied payment on the basis that the service wasn't "necessary," while physicians, who are responsible for ordering most services, are paid "in full." "15 Along similar lines, physicians may admit patients to the hospital and order services for which the hospital may not always receive payment.

Declining or discounted reimbursement rates drive physicians and hospitals to increase volume, which can result in over-utilization and ever higher health care costs. Conceptually, there are good reasons to support reimbursement schemes that put physicians and hospitals at joint risk for cost and quality, but remain incentive-neutral. In practice, this is hard to achieve and remains one of the central challenges of health care reform.

\section{Medical Liability}

The U.S. Department of Health and Human Services estimates that the country spends $\$ 28$ billion annually on medical malpractice litigation and related defensive medicine. ${ }^{16}$ While it is arguable whether Arizona is in the front rank of states experiencing a genuine medical liability "crisis," and while there is debate on the impact and importance of various tort reforms proposed, there is little disagreement across the health care industry that medical liability insurance premiums have surpassed sensible levels and make it increasingly difficult for physicians and hospitals to provide necessary care.

Medical liability and skyrocketing insurance premiums impact physician-hospital relations on multiple levels. Given the excessive premiums, some physicians in "high risk" specialties such as Obstetrics and Gynecology (OB/GYN) and surgery choose to either reduce their exposure to more complicated cases, not practice at all, or migrate to less expensive/less risky settings. Hospitals, in turn, have to deal with the same excessive premium rates and find physicians who are willing to take the necessary cases and assume risk. Ironically, 
physicians and hospitals can end up as adversaries in medical malpractice cases (hospital's "fault" versus the physician's fault), which further drives a wedge in relationships.

The chief problem, however, is the disconnect between the current medical liability system and efforts to improve patient safety and reduce medical error. Both physicians and hospitals are reluctant to be open about errors without adequate legal protection. Where patient safety depends on open communication, transparent information and integrated systems design, the medical liability system fosters secrecy, fragmentation and adversarial relationships. Like other issues that impact physician-hospital relationships, medical liability is grounded in a litigious, regulated and bureaucratic culture of blame, recrimination and redress.

\section{Advancements in Capital and Technology}

In 1970, the U.S. health care industry covered $\$ 73$ billion in economic activity, or seven percent of Gross Domestic Product (GDP). In 2004, health care covered \$1.8 trillion in economic activity, or over 15 percent of GDP. That kind of spectacular growth has not gone unnoticed.

Industry growth has attracted "large, global, medical and infrastructure technology enterprises and specialty services management companies" that bring new sources of capital and expertise to the table. ${ }^{17}$ Where physician specialists in potentially high margin fields formerly had to rely on hospitals to develop the infrastructure necessary for their operational theater, they are now actively courted by a growing list of companies interested in all manner of "joint" ventures to cash in on the boom of procedures and services driving industry growth. Whether it's competition from privately financed ambulatory surgery centers (ASCs) and specialty hospitals or other nonprofit hospitals that create a "hospitalwithin-a-hospital partnership with physicians to gain greater community market share, general hospitals are increasingly at risk for losing significant income from the defection of high-margin specialty groups. Rather than "wait their turn" for hospitals to come up with the capital and technology to accommodate their needs, some specialists choose to do business with groups in other markets.

Advances in medical technology also migrate out of the traditional hospital setting and into doctor's offices and free-standing surgical suites. A large multi-specialty group in Phoenix, for example, recently purchased its own positron emission tomography (PET) scanner because of its potential to increase revenue from Medicare.$^{18}$ It is not unrealistic to forecast robotic surgery devices and other emerging technologies moving out of the hospital and into new operational "theaters" in the years ahead.

The availability of new sources of capital and advances in technology, to the degree that they entice physicians away from traditional inpatient settings, can have a dramatic effect on a hospital's bottom line and drive a wedge in relations between management and physician specialists. Table I, for example, illustrates trends in outpatient surgeries and average annual revenue generated by various physician specialties. These trends suggest continued fragmentation and diversification in physician-hospital relationships. On the other hand, if hospitals themselves continue to consolidate into large, integrated health systems, they will be able to regenerate capital at a size and scale beyond what might be available to disaggregated physician business models, and so continue to attract physician affiliation. 


\title{
Table I: Trends, Growth and Revenue
}

\section{Outpatient Trends ${ }^{19}$}

\author{
Outpatient Surgeries $1980 \quad 15 \%$ of All Surgeries \\ Outpatient Surgeries $2000 \quad 70 \%$ of All Surgeries
}

\author{
II. Ambulatory Surgery Volumes \\ Compound Annual Growth Rates $1981-2007$ \\ $13.8 \%$ \\ Freestanding Clinic \\ (physician-owned) \\ $13.4 \%$ \\ Physician Office \\ $8.1 \%$ \\ Hospital
}

\section{Average Annual Hospital Revenue Generated By Specialty (2004) $)^{20}$}

Specialty
Orthopedic Surgery
Cardiology (non-inv.)
Cardiovascular (inv.)
General Surgery
Neurosurgery
Internal Medicine
Family Practice
OB/GYN
Hematology/Oncology
Pulmonology
Gastroenterology
Psychiatry
Urology
Nephrology
Neurology
Pediatrics

Revenue
$\$ 2,992,022$
$\$ 2,646,039$
$\$ 2,490,748$
$\$ 2,446,987$
$\$ 2,406,275$
$\$ 2,100,124$
$\$ 2,000,329$
$\$ 1,903,919$
$\$ 1,802,749$
$\$ 1,781,578$
$\$ 1,735,338$
$\$ 1,332,948$
$\$ 1,317,415$
$\$ 1,121,000$
$\$ 924,798$
$\$ 860,600$

\section{Clinical Integration and the Quality Movement}

On the other side of external forces driving fragmentation between physicians and hospitals are forces driving integration and continuity of care. While financial exigencies make it difficult to sustain efforts to integrate delivery systems and greater coordination of physician and hospital services in the short term, ${ }^{21}$ pressure on the payer side to reduce costs, increase efficiency and improve health outcomes potentially could change the picture in the longer term.

The movement to improve health care quality calls for greater continuity and coordination of care across providers, especially in anticipation of management of chronic diseases and co-morbid conditions among an older population. To the degree that reimbursement mechanisms are designed to reward quality in the form of integrated protocols, teamwork and patient follow through/tracking, physicians and hospitals will find it mutually advantageous to work more closely together with a shared focus on high quality patient care through the system. 
Federal and state initiatives also call for the integration and delivery of care through seamless and interoperable electronic health information systems. There are legal roadblocks to the greater integration of shared electronic records between physician outpatient and hospital inpatient settings (Stark laws), but there is growing momentum to link up what are now separate systems of care through shared electronic networks. This is another reason physicians and hospitals may work more closely together in the future.

On the cost reduction side, large payers (the federal government and major employers) may look at high volume, "over reimbursed" procedures (imaging services, invasive procedures) and begin to "step on" the margins by pressuring physicians and hospitals to find more efficient ways of delivering demonstrably higher quality outcomes. ${ }^{22}$ This, along with other efforts to reduce costs and improve quality through well designed system integration, may drive payers away from small, independent practices, which will then be motivated to link up with larger integrated systems. It remains to be seen how the divergent forces of fragmentation and integration will play out in the health care marketplace.

\section{Lifestyle}

Another factor affecting relations between physicians and hospitals is the so-called "lifestyle" revolution in medicine and other professions. Compared to previous generations of physicians, engineers and other professionals, who often identified strongly with their profession or affiliated institutions and were totally absorbed in their work, today's young professionals seek more control over their lives and a balance between professional, family, hobbies and other leisure pursuits.

Some believe this is primarily driven by "feminization" of professional culture as more women entering medicine and other professions (close to one-half of today's medical school graduates are women; 25 percent of physicians practicing in Arizona are women, and this will increase in the future), but lifestyle issues cut across gender, race and other social indicators and are making inroads into all aspects of contemporary life in the U.S. and other advanced industrial societies. Today's young professionals identify less with their profession and/or specific institutions like hospitals and corporations and more with themselves as individuals making choices of "enlightened self-interest" among a smorgasbord of "lifestyle" choices. In general, younger physicians today are less concerned with business operations and more concerned with making a personal connection with their work.

This has a number of ramifications for physician-hospital relations. For example, medical students who seek more control over their lifestyle - characterized as off-beeper time - gravitate towards specialties such as Emergency Medicine, Radiology, Ophthalmology, Anesthesiology and Dermatology and away from areas such as primary care, internal medicine and general surgery. Applicants for family practice residencies have declined now for eight years in a row, ${ }^{23}$ which has implications for how patients are referred to hospitals and tracked following discharge. More physicians are unwilling to "take call" on weekends in order to spend more time with their families or engage in other pursuits. Physicians in high margin specialties like Orthopedics and Cardiovascular Surgery are attracted to business arrangements where they can control their schedules and not be at the mercy of a hospital's scheduling of multiple specialties and OR access. Hospital executives report more interest in job sharing, part-time scheduling and work patterns that don't require long hours on site.

The implications of changing professional lifestyles extend well beyond medicine. It's a fascinating subject that we cannot pursue here. 
"It's self-

interest. I'm

not going to

work as hard

for you as I do

for me. I'm not

going to sell

my business

for less than

it's worth."

Arizona Specialist

\section{Dual Cultures}

In the history of physician-hospital relations, much is made of the differences in "culture" between the two groups, or the set of social patterns, values, attitudes, expectations, interests and concerns that define the context of behavior. We review some of these differences, with the important caveat that not all physicians and hospital executives fall neatly into stereotypical role and relationship patterns.

Historically, physician clinicians and hospital managers have adopted dual roles: ${ }^{24}$

\section{CLINICIANS}

Doers

1:1 interactions

Reactive

Immediate gratification

Deciders

Value autonomy

Independent

Patient advocate

Identify with profession

\section{MANAGERS}

Planners, designers

$1: N$ interactions

Proactive

Deferred gratification

Delegators

Value collaboration

Participative

Organization advocate

Identify with Organization

We don't have to review all of the exceptions to this dualistic schemata to appreciate that in terms of culture, physicians are essentially soloists and inwardly driven, and hospital managers are team-oriented and outwardly driven.

- A physician might be willing to spend whatever it takes to help their patient. A hospital manager has to put the expenditure into a larger financial context of competing requests.

- A physician sees patients one at a time. A hospital manager is required to address the needs of multiple patients and meet community health needs.

- For physicians, reimbursement is a personal issue. For hospital managers, it is an organizational issue.

- Physicians may detest meetings and organizational politics. It interferes with practice. Hospital managers may schedule numerous meetings and "manage" the politics. It's their model.

- Physicians are acculturated by years of scientific training and personal judgment informed by experience (eminence-based). Hospital managers are trained to manage large organizational enterprises on sound scientific and business practices (evidence-based).

- Physicians view HMOs and other health "enterprises" as more interested in managing costs than in managing care. Hospital managers know that if there isn't a margin, there can't be a mission.

- Many physicians operate in small groups with local ownership and control. They tend towards the anarchistic. Hospital managers operate in large organizations with diffuse control. They tend towards the bureaucratic.

- Physicians value autonomy and consensus - a guild model. Hospital managers emphasize tight business practices and administrative lines of communication - a corporate model. 


\section{Hospital Best Practices}

Before we turn to the larger question of whether the external factors briefly summarized here will keep physicians and hospitals at arm's length or leverage greater coordination and integration, we highlight emerging best practices, particularly those initiated by hospitals themselves, to foster improved physician-hospital relationships.

\section{Communicate, Communicate, Communicate}

All best practices in improving physician-hospital relationships - all enduring relationships of any kind, in fact - depend on developing trust and mutual reciprocity over time through open and regular communication. The one common ingredient in talking with physicians and hospital executives about their future relations was the importance of listening to each other, having adequate input into decisions, and developing a culture of inclusion.

\section{An Informal Survey}

AzHHA's Building the Bench Leadership Development participants informally interviewed 26 physicians representing diverse specialties and practice settings and asked them to rate the following factors from most important (1) to least important (9) in terms of contributing to better physician-hospital relationships:

1. Nursing competence

2. Regulatory issues

3. Hospital efficiency

4. Income considerations

5. Level of participation in operational decisions

6. Administration's responsiveness to physician concerns

7. ED call coverage

8. Changing medical technologies

9. Threats to clinical autonomy

As Figure I indicates, Arizona physicians believe the prime contributing factors to improving relationships between themselves and hospitals are administrative responsiveness to physician concerns and participation in operational decisions both grounded in ongoing communication. Interestingly, factors such as income considerations, regulatory issues and changing medical technologies were perceived to be less important. Not all physicians are driven by the desire for more money and access to sophisticated technology.

\section{Figure I: Physician's Perceptions of Factors Contributing to Better Relationships Between Physicians and Hospitals}

Administration's Responsiveness

Level of Participation in Operational Decisions

Hospital Efficiency

ED Call Coverage

Threats to

Clinical Autonomy

Nursing Competence

Income Considerations

Regulatory Issues

Changing Medical Technologies
"On my first

day in the

hospital, the

CEO came up, introduced

himself, and said, 'Welcome to the family.' I can't begin to tell you how good that made me feel."

Arizona Hospitalist 
"The hospital

wants you

to check in

three hours

before the

procedure.

It's worse

than Motor

Vehicles.

It's all about

customer

service.

That's why

outpatient

centers are

so popular."

Arizona Specialist

\section{Clinical Quality}

While ongoing and open communication underpins all effective physician-hospital relations, clinical expertise keeps physicians and hospitals together for the long term.

A national survey of over 500 physicians representing 10 specialties by the Clinical Advisory Board ${ }^{25}$ underscored eight major critical indicator categories in the choice of hospitals by physicians for their patients. Ranked from the most important to the least:

1. Physician expertise

2. Nursing competence

3. Hospital efficiency

4. Hospital characteristics

5. Clinical practice support

6. Innovative technology

7. Hospital administration/decision making

8. Income considerations

The expectation of exceptional clinical quality - for both physicians and nurses - is the most important factor in the physician's selection of a hospital. Within this category, consulting and specialist physician expertise rises to the top of the list of preferred hospital characteristics.

There are a number of tactics hospitals might consider to improve specialist recruiting and strengthen specialist focus:

- PHYSICIAN ORIENTATION/SUPPORT. Contributions to transition with liaison, income guarantees, payment of tail malpractice insurance, relocation allowance, assigning preceptor for socialization into the hospital "family" and culture.

- LOAN FORGIVENESS. Repay medical school loans for high demand specialties.

- RECRUITING CAMPAIGN. Provide human resources and marketing support for groups attempting to recruit specialists.

- ED CAll COMPEnSATION. Provide productivity-based or stipend-based pay for specialists on call.

- MAnAgement StIPEndS. Pay high-demand specialists for collaborating on improvement and development efforts.

- REMOTE ACCESS. Facilitate remote reading of radiology exams for selected specialists.

- CONTINUINg MEdical EdUCATION (CME). Host and/or provide support for CME.

\section{Nursing Quality}

Improvements in clinical nursing quality to address physician concerns about nursing staff competency center around rapid development of new hires, continuous staff development and retention of the best staff. Consistent nurse/physician assignments, daily rounding with physicians and physician-specific nursing scripts that detail physician preferences foster more productive teamwork and lead to increased physician satisfaction with nursing services. Of course, to increase nurse satisfaction with physicians, this works the other way round as well. 


\section{"Efficiency is what drives physicians to fewer hospitals, and eventually out of hospitals altogether."}

Arizona Specialist

\section{Operational Efficiency}

In addition to improving clinical quality, commitment to efficiency is an important focus for hospitals that seek to improve physician relations. Efficient operations might include such issues as availability of preferred operating room (OR)/Cath lab times, availability of anesthesia, timely execution of diagnostic and medication orders, ease of scheduling outpatient appointments and consistent availability of inpatient beds.

Access and turnaround times can be targeted with specific process focus:

- BLOCK SCHEDULING. Allocate blocks both to physician groups and individual physicians. Times determined by a medical staff committee that examines utilization history on an ongoing basis.

- PREOPERATIVE PREPARATION. Centralize diagnostic evaluation, involve anesthesia, eliminate unnecessary steps.

- OR TURNOVER. Standardize instruments by procedure, evaluate sterilization time required, monitor environmental services clean time.

- MEDICATION ADMINISTRATION. Evaluate computerized medication order entry and unit-based computerized dispensing.

- CALL CENTERS. One telephone number to call for outpatient appointments.

- DIAGNOSTIC TESTS. Consider radiologist incentives for accuracy and improved speed; develop models to improve diagnostic lab efficiency and speed.

- INPATIENT BED AVAILABILITY. Develop models to increase "throughput" with increased inpatient bed availability. ${ }^{26}$

While there is much that hospitals can do to improve clinical quality and operation efficiency, the fact remains that the diversity and complexity of cases that find their way into hospitals - the "court of last resort" for medical care - make it difficult for them to reach the efficiency standards of freestanding surgery centers. Physicians will continue to migrate out of hospitals - the issue is whether, and how, hospitals will change facility, service and governance design to accommodate them. 
"The

telephone

doesn't work

anymore.

People are

too busy.

We need

a seamless

electronic

medical

record.

That would

go a long

way to

making both

physicians

and hospitals

more

productive."

Arizona Physician

\section{The Promise of \\ Health Information Technology (IT)}

Access to the latest medical technology is less important to physicians in establishing strong relationships with hospitals than perceptions of administrative responsiveness, clinical excellence and shared decision making. On the other hand, infrastructure and equipment that support high-quality patient care, such as electronic health records (EHRs), virtual imaging and electronic order entry, are consistently cited by physicians as holding the potential to "have a significant impact on their success." 27

Until recently, only a few hospitals have funded community physician IT investments and operations "due to fraud, abuse and Stark prohibitions." ${ }^{28}$ This is liable to change as the federal government promotes the development of new regional health information organizations (RHIOs) to create the efficient exchange of health care information between physicians, hospitals, other providers, health plans and consumers. ${ }^{29}$

Many hospitals have learned the hard way how difficult and expensive it can be to develop internal health information systems, let alone systems that link up local physician and outpatient settings. Nevertheless, health IT is a "sticky" technology to the extent that physicians who invest in a particular hospital's IT system tend to stay with it and not jump ship to competing systems. Large medical groups and provider-based health plans like Kaiser Permanente have installed EHRs and reaped the efficiencies and patient satisfaction rewards of improved IT; the question now is how quickly independent hospital and physician practices will follow suit.

There are numerous challenges, but the biggest one is funding. Hospitals lack the capital to install broad-based community IT systems on their own, and physician practices are reluctant to invest in community IT if they see the benefits of that investment reaped by health plans, employers, pharmacies, labs and patients, and not by them directly.

Community health IT will be done collaboratively, or it won't be done at all. In the words of one observer,

"Hospital leaders bring a great deal to the table... They have IT, quality improvement and medical informatics staff that can help support the installation and shared use of new data systems to improve health care across their community. They have missions and long histories of community service... How they participate in the next phase of community information technology networks will play a large part in determining the future of hospital-physician relations. " ${ }_{30}$ 


\section{Own, Rent or Share: Physician-Hospital Alignment Models}

Facing reimbursement pressures and perceptions of increasing intrusion into the physicianpatient relationship by health plans and regulators, physicians will continue to seek more control, autonomy and financial reward in new practice configurations. In the future, as now, physicians will either be active partners with hospitals or compete against them. There will be few left hanging in the middle.

Realizing this, hospitals can employ a number of strategies and models to optimally structure relationships with physicians: employ them directly, utilize various joint venture/ contractual models for selected services, or structure other "shared" clinical, administrative and governance systems.

Amid the various and often bewildering approaches to aligning physician and hospital interests, made even more complicated by a forest of legal restrictions, it's no surprise that there is little agreement on the best course to follow, which varies according to location, size, resources, practice patterns, history and entrenched interests. Indeed, the white papers developed by AzHHA's three leadership development teams for this project came to different conclusions about the best way to align physician-hospital interests. What makes sense for a large urban hospital administrator does not necessarily seem the best solution for a hospital in a smaller community.

With that caveat, we briefly summarize general approaches to physician-hospital alignment.

\section{Employment Models}

One approach is for hospitals to employ physicians directly. As employees, they work assigned shifts, receive a base salary (often with incentives tied to various quality, production and patient satisfaction metrics), and participate as full members of the hospital medical staff in all areas related to governance, clinical quality, operational efficiency and continuing education and improvement.

In this arrangement - which has a multitude of variations in actual practice - the hospital assumes the cost and management of employing the physician, including benefits, malpractice insurance, office space and billing for professional services. For a busy hospital in a competitive, relatively unregulated market like Arizona, where physicians have plenty of work and choices to practice in well-financed specialty and outpatient settings, often the most immediate and direct solution to a shortage of skilled professionals is to have them on staff and give them the freedom to practice medicine without the hassles of running their own businesses in outpatient settings and dealing with oppressive overhead costs (50-60 percent in smaller shops) and paperwork. For some physicians, this is an attractive proposition. 


\section{The Rise of the Hospitalist}

The field of hospital medicine continues to grow. From a few hundred "hospitalists" in the mid 1990 - physicians who provide continuity of care exclusively in a hospital setting - numbers increased to more than 8,000 by the end of 2003 and are expected to triple to 25,000 by $2010 .^{32}$

Most hospitalists come out of internal medicine training, with other specialties such as family practice, pediatrics and pulmonary medicine also represented. Reasons for choosing a hospitalist career vary, but many prefer to practice inpatient medicine exclusively, enjoy the flexibility of scheduling and predictable practice patterns, and feel less hassled by outpatient business concerns. In addition to providing care for patients whose admitting physicians prefer not to practice in hospitals or lack admitting privileges, hospitalists may “increasingly substitute for intensivists in ICUs, team with subspecialists to care for complicated patients, function as primary attending physicians in skilled nursing facilities and care for nursing home patients hospitalized at night." ${ }_{33}$

One of the keys to a successful hospitalist program is the level and quality of communication between the hospitalists and the referring outpatient family practitioners and specialists.
Arizona hospitals are turning to hospitalists to provide prompt, attending physician services, $24 / 7$ on-site coverage and improve services for outpatient admitting physicians. Ideally, this can result in more efficient use of hospital resources, better throughput, and improved patient care and satisfaction all the way round. Hospitalists help to address unassigned patients due to physician shortages and, in Arizona at least, the large number of seasonal winter visitors without a local primary physician. On the downside, there can be problems with discharge and follow through, lack of good communication between inpatient staff and admitting physicians, and all of the financial, cultural and administrative challenges of "managing" on-site physicians. Another potential downside is patient dissatisfaction when dealing with multiple providers during a single stay. One Arizona hospital, for example, experienced a 20 percent decline in physician ratings over a two-year period during which a hospitalist model was implemented.

Some hospitals choose to build their own hospitalist program, while others choose to contract out hospitalist services to local, regional or even national groups, including specialist groups. There are various pros and cons to any approach, ${ }^{34}$ and each hospital has to determine the best way to "exert contractual control over performance and quality." Based on interviews with Arizona physicians and hospital administrators, one of the keys to a successful hospitalist program is the level and quality of communication between the hospitalists and the referring outpatient family practitioners and specialists. Local, home-grown hospitalist groups often have these personal connections and relationships, while larger outside groups do not. 


\section{Potential Advantages}

- Alignment of physician (medical staff) and hospital needs in a common setting

- Higher physician job satisfaction

- Improved practice management, continuity and quality of patient care

- Autonomy (relative) over clinical practice

- Leverage for negotiating managed care contracts

- Legal support for creating internal "centers of excellence"

\section{Potential Disadvantages}

- "Owning" physicians can be expensive and administratively challenging ${ }^{31}$

- Problems with creating economies of scale through practice consolidation

- Failure to develop proficient practice management expertise

- Physicians may lack ability to add profitable ancillary/diagnostic services to their practice

- Cultural/practice difference between participating physician partners

- "Handoff" problems with discharge/follow-up

- Failure to integrate high dollar volume specialists

\section{Joint Venture (JV) Models}

Joint ventures - generally outpatient arrangements where hospitals and physicians share in the equity and/or management of a separate "service line" entity - present other options for aligning physician-hospital interests. Common examples include ambulatory surgical centers (hospital and surgeons), endoscopy centers (hospital and gastroenterologists), imaging centers (hospital and radiologists), cardiac cath labs (hospital and cardiologists), and radiation therapy centers (hospital and radiation oncologists).

Some JVs are equity-based, where the hospital and selected physicians share the capitalization of the venture, usually resulting in a limited liability or limited partnership company that is separate from both the hospital and physicians. Typically, the hospital is the "majority" partner, with 51 percent or more of the investment. These JVs can enroll as separate Medicaid and Medicare providers, contract separately with commercial payers, and employ their own staff or lease staff from other sources. Physician equity partners often have significant control of day-to-day operations and, as co-investors, earn a return if the venture is successful.

Other JVs might be structured as contractual models. ${ }^{35}$

- LEASING. Physician groups lease equipment, space and possible technical personnel from a hospital or hospital-physician JV on a time-share basis.

- PURCHASED SERVICES AGREEMENT. The hospital purchases services from a separate third party, which are then treated and billed as hospital services. The third party might be a specialty physician practice or a separate hospital-physician JV.

- CLINICAL CO-MANAGEMENT. Under a management service agreement, the hospital contracts administrative responsibilities for an outpatient service to a physician-owned management company.

- “GAINSHARING." Hospital and physician groups enter into agreements to "share" in cost reductions achieved through improved clinical coordination, treatment and management. 


\section{"Our group}

asked the

hospital if

they wanted

to be a

partner in

establishing

an outpatient

endoscopy

center, and

they said sure,

but we have

to have 51

percent

ownership.

Needless to

say, we built

it ourselves."

Arizona

Gastroenterologist
Space does not permit consideration of the various legal, financial and managerial issues that complicate JVs. Suffice it to say that JVs are a growth area in the legal industry.

\section{Potential Advantages}

- Aligns physician-hospital interests, decreases competition and duplicate services

- Improves physician recruitment, decreases risk of physician defection

- Increased operating efficiency and physician satisfaction

- Higher returns (conceivably) for both parties

- Greater patient satisfaction levels

- Shared control and management responsibilities

\section{Potential Disadvantages}

- Can result in decreased revenue for all parties if not structured correctly

- Reimbursement for outpatient services can be lower than identical inpatient services

- Can set up a "me, too" precedent for other physicians/staff related to hospital

- Major legal considerations that can affect hospital's nonprofit status

- Relatively high risk of failure, based on history

- Level of physician-hospital trust often not sufficient for long-term investment

- Fragmentation of a hospital's service lines through separate JVs could prove unacceptable

- Typically benefits specialists more than generalists

\section{Other Models}

\section{Participating Bonds}

Participating bond transactions (PBTs) present an alternative to equity joint ventures for nonprofit and public hospitals. Whereas traditional tax-exempt hospital bonds pay investors a fixed low interest rate, "participating" tax-exempt bonds pay investors rates that can be two to three times higher. That's because PBTs are more risky than traditional bonds. They have no public market and are typically sold as subordinated instruments. Typically, PBT financing of joint ventures "does not require the parties to bear higher operating costs and regulatory risks. ${ }^{36}$ Further, they pay investors with tax-exempt interest based on the economic performance of the joint venture.

Primary care physicians, who are rarely invited to invest in any specialist-owned facility, may appreciate the opportunity to supplement their incomes with a PBT investment. Some primary physicians may resent the fact that specialists' income is higher than theirs - and will get even higher when specialists invest in a medical facility that will make money only if the primary care physician continues to refer. ${ }^{37}$ PBTs are one way for them to participate.

On the other hand, while PBTs may appear attractive to nonprofit hospitals and groups such as primary care physicians, they might be less attractive to specialists who seek maximum return for their efforts through equity JVs structured to their advantage - or to own the outpatient center outright. No one model of aligning hospital-physician interests, it turns out, appeals to everyone. 


\section{Clinical Councils}

Some hospitals, faced with lack of physician involvement in clinical, management and strategic planning decisions, choose to establish clinical councils, a shared leadership model through which appointed members of the hospital's medical staff serve as advisors to the hospital CEO and have significant input into all clinical and administrative matters.

For example, a physician leader from each "Center of Excellence" in a hospital might serve on the council and, together with his/her peers, have responsibility for performance improvement, quality standards, finance, facilities development and equipment purchasing, information services, ambulatory services and medical directorships. This leaves the broader medical staff with responsibilities for department activities, medical staff policy, credentials and peer review. The success of this model - the success of all physicianhospital alignment models, really - depends on open communication and a true partnership approach to decision making. The difficulty lies in resolving the conflicting perceptions of what a "partnership" really is - or ought to be - in a complex organizational system where the financial incentives and cultural orientation are not usually aligned.

\section{Global Payment}

Global payment - defined as one payment for hospital and physician services that is then "split" between the various parties according to mutually selected criteria is touted by some as the best way to ensure alignment of physician-hospital interests. Global payment can be structured within a hospital or implemented by CMS and other payers for hospital-based billings. Some studies show that, done correctly, global payments can achieve a significant reduction in costs and improve outcomes. When physicians have a financial stake in hospital costs, they are more likely to work with administrators to reduce them while keeping quality high. ${ }^{38}$

There are, of course, numerous complications: dealing with the "outlier" cases in any hospital that have multiple complications and specialists involved, problems with data collection and billing services, "gaming" the system (shifting costs to other settings such as home health or skilled nursing), and the usual legal concerns. A full analysis of global payment approaches is outside the scope of this brief report, but we highlight it as an area worthy of consideration. 
"The worst

that I see in

medicine is

driven by pure

entrepreneurial

motivation."

Arizona Physician

\section{Policy Considerations}

Health care has become a vast industry, but one that, at its core, retains the ethical imperative of a social good. A person who shows up at the hospital emergency room at 2 a.m. with a heart attack is not looking to purchase a commodity or have a "branding" experience. She is in terror and fears for her life. Skilled medical professionals will do whatever it takes to help her. Few of us would choose to live in a society where this moral imperative to aid the sick and injured did not exist. ${ }^{39}$

In that context, policy makers and the general public have a major stake in hospitalphysician relationships. Over 80 percent of hospitals in the U.S. are nonprofit, communitybased institutions, and as such they are legally and ethically required to provide necessary medical care to community citizens - a mandate that is impossible to discharge without physicians, nurses and other highly skilled professionals.

\section{Two Scenarios}

SCENARIO I Under one scenario, economic forces and technological developments will continue to motivate physicians to practice in outpatient, "just-in-time" settings, with an emphasis on better reimbursement, greater clinical and service control, mobile and highly specialized hardware/software, improved consumer convenience, speed and efficiency. Over time, the cream will spill off into new, rich vessels, leaving hospitals with the sickest, most marginalized and lowest income patients that no one else wants to treat. Financial and labor pressures will force many hospitals to close their doors. Those remaining will be large, integrated facilities that will cover wider service areas and be highly regulated, much like public utilities. Core safety net and "public" services will be provided under more centralized arrangements. The great majority of physicians will operate as independent, economic actors. Hospital-based physicians will be specialists in their own right; most will be public utility employees.

SCENARIO II Under another scenario, payer pressure to reduce health care costs, increase efficiency and improve quality will drive greater, not less, integration between physicians and hospitals. The proliferation of point-to-point electronic health records, information transparency and new "shared" reimbursement mechanisms will unite hospital managers, physicians and other health care providers under one large electronic grid with multiple local area networks, each characterized by rich knowledge and practice exchanges. More consumers will interface with hospitals and physicians directly, bypassing third party payers, which will morph into information brokers. Hospital managers and physician leaders will develop new ways of working together in powerful synergistic partnerships. More physicians will become managers of sophisticated care delivery teams. Hospital managers will become adept at new product development and integrated systems design. Hospitals will be hubs of extended community health networks.

The future is likely to be neither as stark nor tidy as these scenarios suggest. We can work together to create a desired future, but we can't predict the outcome.

Strong, mutually productive relations between hospitals and physicians are vital to a strong Arizona and an accessible, affordable and high quality health care system. To achieve that goal, policy and health care leaders should consider the following: 
- REEVALUATE LAWS GOVERNING PHYSICIAN-HOSPITAL RELATIONSHIPS. Stark, antikickback, anti-trust, tax-exemption and EMTALA laws were promulgated to address specific issues and situations that have since evolved in a contentious and rapidly moving health care industry. There is much that is good and needs to be retained, but there are also places where laws might be adapted to allow for the right kind of physician-hospital integration in the service of more equitable, affordable and quality health care.

- REFORM THE MEDICAL LIABILITY SYSTEM. In our opinion, tort reform is insufficient by itself to improve clinical performance, persuade more physicians to practice in potentially high risk cases and settings, and improve hospital-physician relations. We need a fundamentally different medical liability system that allows openness, transparency, cooperation, teamwork and safety. Hospital and physician leaders need to develop some powerful new models and come to the table prepared to advocate together for them.

- IMPLEMENT A STATEWIDE ELECTRONIC HEALTH INFORMATION NETWORK. TO improve connections between physicians, hospitals, payers and consumers, we need an interoperable, point-to-point information system focused on improving clinical efficiency and safety, improved health outcomes and consumer ownership and involvement in their own health care. In our opinion, the best way to get this done is through a robust public-private partnership. Physician and hospital leaders need to come to the planning table now. The Governor's Health-e-Connection Roadmap Task Force is a place to start.

- EXPERIMENT WITH REIMBURSEMENT APPROACHES. Nothing gets someone's attention like paying them. A system that can reward different providers for both restricting and increasing care at once will never foster integration, cooperation and improved care. Pay-for-performance mechanisms, global payments, quality improvement initiatives all of these are fraught with difficulties, but moving forward with such activities is absolutely vital if we are ever to align system incentives.

- DEVELOP HEALTH CARE KNOWLEDGE AND PRACTICE NETWORKS. Large, successful global companies spend significant resources and time on developing internal knowledge and practice networks, where information and ideas flow freely in open communication to create opportunities for shared success. These are not clinical councils, "partnerships," joint ventures or advisory groups. They are horizontal networks of both structured and unstructured communication - feedback loops - where participants develop ideas, connections, collegiality and shared success over time. All successful systems biological, mechanical, organizational - depend on these loops. Hospital and physician groups can build them together. They provide a tangible forum for promoting leadership, partnership and proactive change.

- REDEFINE AND EXPAND THE CONCEPT OF PARTNERSHIP. The rhetoric of partnership and collaboration often masks an imbalance of power and mutual distrust between the parties. As any trained counselor knows, the first thing one does in a dysfunctional marriage is to bring those differences out in the open and candidly talk about them. That's where all good relations start. A partnership is 50/50, not 51/49. Further, in today's health care industry, a successful partnership between physicians and hospitals will depend not only on their own respective involvement in strengthening the marriage, but also in bringing in the other parties to the dispute, namely other health professionals, health plans and consumers. Some physicians interviewed for this policy primer suggested that hospital-physician partnerships might be structured differently if they were mediated by trained third parties rather than entered into without an independent and neutral "filter." 
Finally, in interviewing hospital and physician leaders about their relationships, we were struck by how most agreed that the health care industry was, in the words of one physician, in danger of "losing its soul," and how there was more to this issue than just making more money and looking out after Number One. Hospital administrators are acutely aware of their larger community responsibilities and roles, and many physicians are bothered by the increasing industrialization of medical practice, and wish to spend more time with patients and improving medical care. We found this encouraging.

The forces pulling apart physicians and hospitals are strong and real enough, but so are the forces of greater integration, improved quality and efficiency and, ultimately, the human spirit and hope. Can this marriage be saved? We think so.

\section{Arizona Hospital and Healthcare Association 2005 Building the Bench Leadership Development Class}

Vicki Began, BSN, MSN

Director, Women and Children's Services

University Medical Center, Tucson

Mike Campbell, BS, ADN

Chief Executive Officer

Arizona Orthopedic Specialty Hospital

Robert Dent, MBA/HCM, BSN, RN, CHE

Administrator, Sterling Regional MedCenter

Banner Health, Sterling, Colorado

Debra Flores, $M B A, B A$

Chief Operating Officer

Paradise Valley Hospital

Sharon Gardner, MPA, BA, SPHR

Vice President, Human Resources

Yuma Regional Medical Center

Troy Garland, MBA, BA

Assistant Director of Nursing

Chandler Regional Hospital

Sue Hanauer, MSN, BSN

Vice President, Patient Care Services

John C. Lincoln Hospital-North Mountain

Brian Hoefle, MA, BA

Chief Financial Officer

Navapache Regional Medical Center, Show Low

Jayleen Jensen, MBA, BSN, RN

Executive Director, Faculty Operations

St. Joseph's Hospital and Medical Center
Laura McKay, MBA, BS, AAZ

Director of Medical Imaging

Maricopa Integrated Health System

Lisa Mead, MS, BSN, CPHQ

Director, Quality and Organizational

Effectiveness

Scottsdale Healthcare

Joseph Anthony Moncayo, MBA, BSRT, (R) (CV) (AART)

Vice President, Ancillary Services

Casa Grande Regional Medical Center

Vikki Noyes, MBA, BS

Associate Administrator

Banner Thunderbird Medical Center

Dina Rojas-Sanchez, ME, BA

Assistant Administrator

Carondelet Holy Cross Hospital, Nogales

Marj Sisson, BS, MBA

Director, Transition Management

University Medical Center, Tucson

Lois Uniat, MHS, BSN

Director, Managed Care/Care Management

Scottsdale Healthcare

Laurel VanHalderen, MHA, BS

Administrator, Mental Health

and Behavioral Science

Carl T. Hayden VA Medical Center 
1 Stevens, R., In Sickness and Wealth: American Hospitals in the Twentieth Century, New York: Basic Books, 1989, p. 7.

2 Ibid., p. 87.

3 Quoted in Ibid., p. 241.

4 Quoted in Ibid., p. 244.

5 This is the familiar "no margin, no mission" argument. Hospital administrators might paint a softer gloss, but the industry has been bottom-line driven for some time now.

6 These models are taken from the work of L. Casalino and J.C. Robinson in "Alternative Models of HospitalPhysician Affiliation as the United States Moves Away From Tight Managed Care, The Milbank Quarterly, Vol. 81, No. 2, 2003, pp. 331-351.

7 Ibid., pp. 332-333.

8 Ibid., p. 335 .

9 Adapted from McGowan, R., Strategies for Strengthening Physician-Hospital Alignment: A New England Study, a Bristol Group Mitretek white paper, September 2003, p. 16.

10 Adams, D., “Physicians are Working More, Enjoying it Less," American Medical News, June 2002.

11 Intellimed International Corporation, “Arizona Physician Need Assessment Report," February 28, 2005, prepared for Casa Grande Regional Medical Center. We have noted elsewhere the problems associated with the linear extrapolation of physician supply in Arizona (Lockhart, C., Hughes, R., Dancing on a Pin: Health Planning in Arizona, St. Luke's Health Initiatives, November, 2005), but the general point of shortages is defensible in the current context.

12 Learn more about Stark law and compliance at www.starkcompliance.com.

13 Zismer, D., "Physician Relations: The Achilles Heel of Hospital CEOs," Discovery (Dorsey Health Strategies Group), Feb. 2003, p. 6.

14 Ibid.

15 Goodroe, J., Andrews, T, “Physician/Hospital RiskSharing, www.goodroe.net/risk_sharing.html.

16 Iglehart, J., “The Malpractice Morass: Symbol of Societal Conflict," Health Affairs, 23:4, 2004, p. 7.

17 Zismer, op. cit., p. 5 .

18 Lesser, C., et. al, “Initial Findings from HSC's 2005 Site Visits: Stage Set for Growing Health Care Cost and Access Problems," Center for the Study of Health Systems Change Issue Brief, No. 97, August 2005.

19 American Medical News, April 15, 2002.

20 Merritt, Hawkins and Associates, 2004 Survey of Physician Inpatient/Outpatient Revenue.

21 See Lake, T., et. al, "Something Old, Something New: Recent Developments in Hospital-Physician Relationships," Health Services Research, 2003 Feb. 38, pp. 471-88.
22 Zismer, D., "Why Will the Future of Not-For-Profit Healthcare Delivery in the U.S. See More, Not Less, Integration of Hospital and Physician Interests? The Top 10," Dorsey Health Strategies Discovery, November/December 2004, www.dorsey.com.

23 AMedNews, April 4, 2005.

24 Currey, W., ed., The Physician Executive: An AAMD Publication, June, 1988, p. 66.

25 Clinical Advisory Board, Recovering Physician Loyalty: Lessons on Crafting a True HospitalPhysician Partnership, The Advisory Board Company, Washington, DC, 2002.

26 Improvements in diagnostic tests and inpatient bed availability are, according to hospital administrators who helped to develop this report, concepts that need more development. National initiatives like Urgent Matters (www.urgentmatters.org) focus on improving clinical and operational efficiency through better "throughput" between emergency rooms, ORs and access to inpatient beds.

27 Messigner, S., Welter, T., “Today's Key Drivers of Physician Loyalty," Healthcare Financial Management Association, May 2003, www.hfma.org.

28 Coye, M., “Unplugged? Hospital-Physician Relations in the New World of IT," www.hospitalconnect.com/hhnmostwired, March 3, 2005.

29 This is the force behind Arizona Governor Janet Napolitano's September 2005 Executive Order to develop a roadmap for the creation of a statewide electronic health information exchange.

30 Coye, op. cit.

31 Many hospitals that purchased or bought into physician practices in the era of tight managed care in the mid1990 learned the hard way how expensive it can be.

32 Vernick, R., Wilson, M., "The Rise of Hospitalists, Part I," Hospital and Health Networks Magazine, August 23, 2005, www.hhnmag.com.

33 Quoted in Ibid.

34 Pros and cons of hospitalist models are discussed in Vernick, R., Wilson, M., "The Rise of Hospitalists, Part II," Hospital and Health Networks Magazine, August 30, 2005, www.hhnmag.com.

35 Cohen, R., Rock, K., “Physician-Hospital Joint Venture and Affiliation Models: Planning, Legal and Structuring Considerations," www.kutackrock.com/publications

36 PBTs are complicated and beyond the scope of this paper. See "Participating Bond Transactions: An Important New Alternative to Equity Joint Ventures," McDermott Will and Emery, August 13, 2003. www.mwe.com.

37 "Physician Competition: Implications for Hospital Boards," Environmental Scan - Trends and Responses for Great Governance, 2005-2010, The Governance Institute, pp. 31-35.

38 Goodroe, J., op. cit.

39 We distinguish between health care as an industry and health care as a social investment more fully in Lockhart, C., Hughes, R., Dancing on a Pin: Health Planning in Arizona, St. Luke's Health Initiatives, November 2005. 


\section{Our Mission}

To improve the health of people and their communities in Arizona, with an emphasis on helping people in need and building the capacity of communities to help themselves.

For a complete list of Arizona Health Futures publications, conferences and other public education activities, visit the SLHI web site at www.slhi.org. If you would like to receive extra copies of a publication or be added to our mailing list, please call 602.385 .6500 or email us at info@slhi.org.
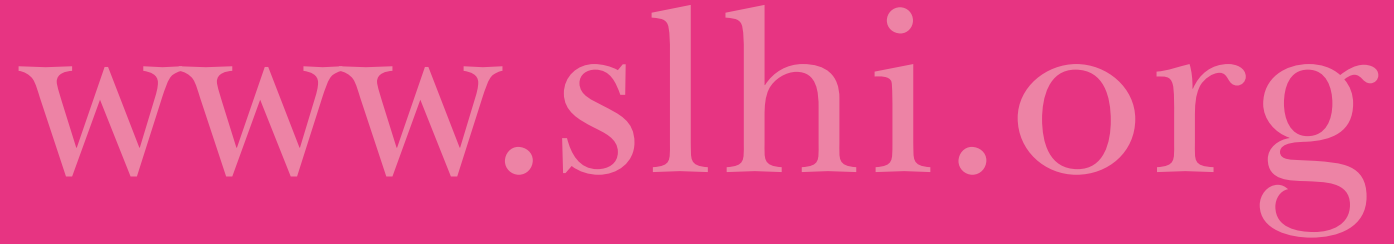

St. Luke's Health Initiatives is a public foundation formed through the sale of the St. Luke's Health System in 1995. Our resources are directed toward service, public education and advocacy that improve the health of all Arizonans, especially those in need.
Analyst:

Roger A. Hughes, Ph. D.

Graphic Design:

Chalk Design

(C) 2005

All Rights Reserved.

Material may

be reproduced

without permission

when proper

acknowledgment

is made.

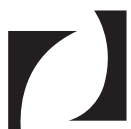

St. Luke's Health Initiatives

A Catalyst for Community Health

\section{PLEASE NOTE} OUR NEW ADDRESS

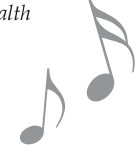

2929 N Central Avenue

Suite 1550

Phoenix Arizona 85012

www.slhi.org

info@slhi.org

602.385 .6500

602.385 .6510 fax 